\title{
X-ray monochromator system for use with synchrotron radiation sources
}

\section{Citation}

Golovchenko, J. A., Levesque, R. A., Cowan, P. L. 1981. X-Ray Monochromator System for Use with Synchrotron Radiation Sources. Review of Scientific Instruments. doi:10.1063/1.1136631.

\section{Published Version}

doi:10.1063/1.1136631

\section{Permanent link}

http://nrs.harvard.edu/urn-3:HUL.InstRepos:29407055

\section{Terms of Use}

This article was downloaded from Harvard University's DASH repository, and is made available under the terms and conditions applicable to Other Posted Material, as set forth at http:// nrs.harvard.edu/urn-3:HUL.InstRepos:dash.current.terms-of-use\#LAA

\section{Share Your Story}

The Harvard community has made this article openly available.

Please share how this access benefits you. Submit a story.

\section{Accessibility}




\title{
X-ray monochromator system for use with synchrotron radiation sources
}

\author{
J. A. Golovchenko, R. A. Levesque, and P. L. Cowan a) \\ Bell Laboratories, Murray Hill, New Jersey 07974 \\ (Received 18 September 1980; accepted for publication 4 January 1981)
}

\begin{abstract}
A double crystal $\mathrm{x}$-ray monochromator system has been developed for use on synchrotron radiation sources. The system consists of two separate Bragg reflecting crystals constrained by a mechanical linkage system enabling it to tune continuously in $\mathrm{x}$-ray energy, always maintaining an exit beam of constant offset and direction relative to the incident beam. Accurate parallelism between the two crystals is maintained by a piezoelectrically-controlled analog feedback circuit.
\end{abstract}

PACS numbers: $07.85 .+\mathbf{n}$

\section{INTRODUCTION}

In recent years a great interest has developed in the use of synchrotron radiation from high-energy electron storage rings for atomic and solid state physics investigations. In the $x$-ray region of the electromagnetic spectrum the synchrotron radiation source offers high brightness in an essentially white spectrum with attractive polarization properties. These features coupled with a suitable monochromator scheme and beam optics provide experimentalists with a powerful new tool to probe matter. ${ }^{1}$

Monochromators covering the energy range from a few kilovolts and up generally use single crystals of silicon or germanium as energy dispersive elements. The actual energy band pass of such a crystal is usually determined by combining Braggs' law of crystal reflection with the angular divergence of the beam emerging from the synchrotron. After a preliminary Bragg reflection selects an energy of interest, the beam is often redirected into its original direction by a second reflection off the same crystal planes on a second crystal. This keeps the position of the selected beam at a point several meters from the monochromator from varying wildly as the beam energy is changed. It also keeps the unwanted diffusely scattered $x$ rays from the first crystal from entering the experimental region. Constraints on the parallelism of the first and second crystals in this mode of operation is determined by the angular emittance and acceptance of the crystals used. Values of the order of one-twenty arcseconds are typical.

To meet this constraint the first and second crystals may actually be part of the same crystal, i.e., a groove is "milled" in a piece of silicon with one side of the groove serving as the first crystal and the other as the second. The planes of these crystals are parallel by virtue of the uncut crystalline material connecting them.

Although this and related schemes ${ }^{1,2}$ have been used successfully at synchrotron sources, we have been motivated for several reasons to consider another approach to this problem. Our design objectives were the following: (1) To devise a scheme where each crystal element could be changed easily by the experimenter.
For example separate first and second crystals of different materials, atomic planes, and asymmetries could be picked by the user to suit his needs. This approach eliminates the requirement of elaborate crystal sculptures for different kinds of experiments. It allows for fine tuning of the relative angle between first and second crystal for harmonic rejection purposes or to eliminate detuning due to first crystal thermal loading (a problem anticipated in the new generation of synchrotron sources to appear shortly). (2) To obtain not only a constant output direction, but also a constant beam position (or offset) as the energy is varied. This position was envisioned being of the order of $15-100 \mathrm{~cm}$ displaced from the plane of the synchrotron electron orbit to provide isolation of adjacent experimental ports and expanded possibilities for new experimental ports in the vertical direction. The obvious advantage of constant beam height during energy scanning experiments is also desirable, particularly when large vertical beam displacements are required. (3) To make the central ray of the selected beam impinge at the same point on all monochromator crystals independently of the chosen energy. This feature makes the requirements on monochromator crystal size independent of monochromator size. Extended energy tuning, even with relatively small crystals, can then be envisioned without changing performance due to crystal inhomogeneities or bending. The range of suitable crystals for monochromator use should be extended as a result.

The above design objectives have been met by devising and constructing: (1) a basic mechanical linkage capable of keeping the two crystals parallel within $\sim 30$ arcseconds over an extended energy range with a constant 6 in. offset in beam height. (2) A "corrector" assembly containing a piezoelectric element, which when driven by appropriate electronics reduces angular errors in crystal parallelism of up to 100 arcseconds to tolerable values $(\ll 1$ arcsec). (The crystals mount on the "corrector" and the corrector mounts on the linkage). (3) An electronic feedback system that samples the output beam intensity senses angular drifts out of parallelism between the two crystals, and provides electric drive to the corrector to eliminate it. 


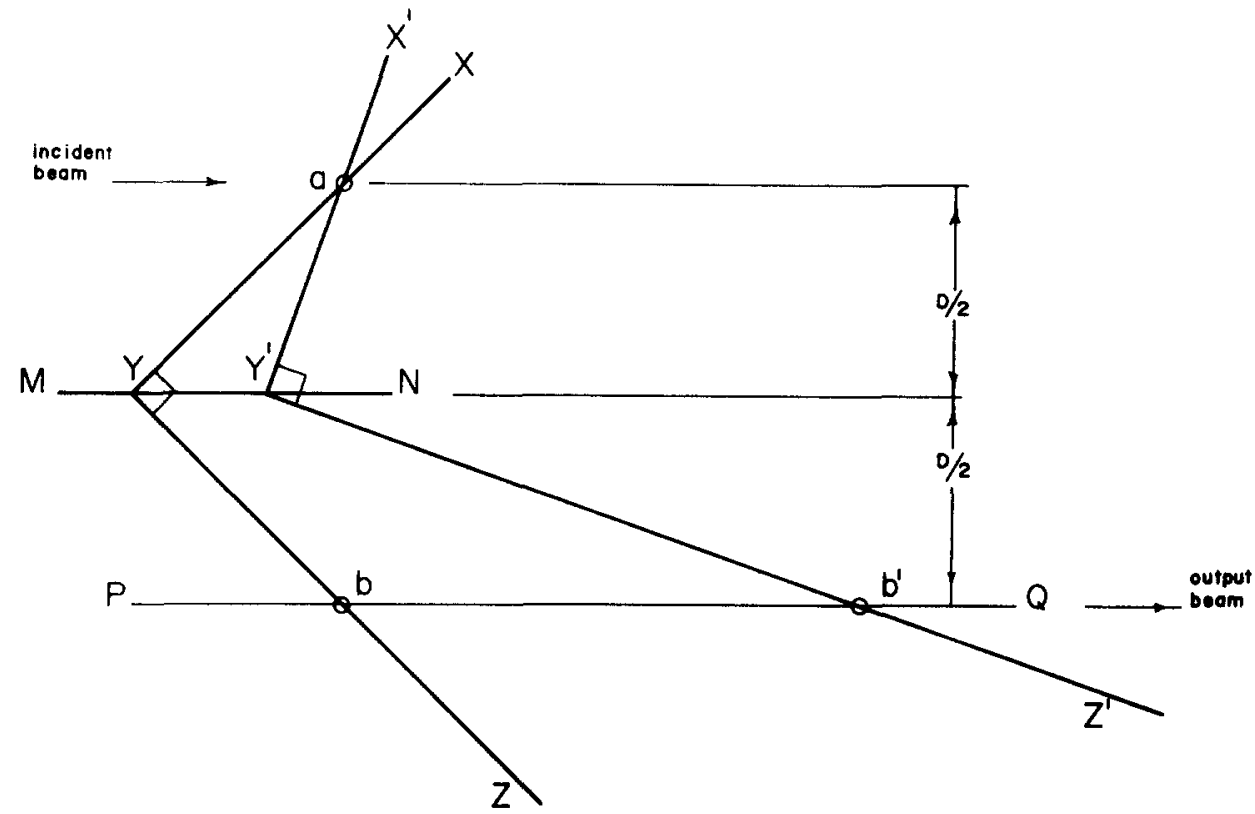

FiG. 1. Geometrical construction itlustrating sliding and rotating motion of rigid right angle. Incoming and outgoing beam heights are indicated. Crystal positions will ultimately be at positions " $a$ " and " $b$."

\section{THE MECHANICAL LINKAGE}

To see the basic principle upon which the monochromator works imagine a rigid right angle made up of bars $Y X$ and $Y Z$ (Fig. 1). We shall require that the position of this right angle in space satisfies the following constraints. (1) The apex of the right angle must always have a vertical height exactly halfway between the height of the beam incident on the monochromator and the desired output beam height. (2) The bar $Y X$ must always pass through some fixed position " $a$ " at the incident beam height. Thus for any given position of the apex $Y$ along the midline $M N$, the rigid right angle must rotate about its apex $Y$ in order that the length $Y X$ intercept the fixed point " $a$ " at the beam height. A second pos- sible position for the right angle consistent with the above mentioned constraints is indicated by $X^{\prime} Y^{\prime} Z^{\prime}$. This sliding and rotating motion of the right angle is the heart of our monochromator and we shall see that it acts as mechanical analogue computer generating proper crystal angles and displacements for our double crystal monochromator.

Next let the first crystal of the monochromator be constrained to be at position " $a$ " with the normal to the reflecting planes " $n$ " required to be along direction $X Y$ (see Fig. 2). Depending on the planar spacing and angle of incidence $\theta$ an energy will be selected by Braggs' Law and this beam will be deflected by $2 \theta$ at " $a$." Clearly different positions of the apex $Y$ along $M N$ will yield different values of $\theta$ and thus of selected energy.

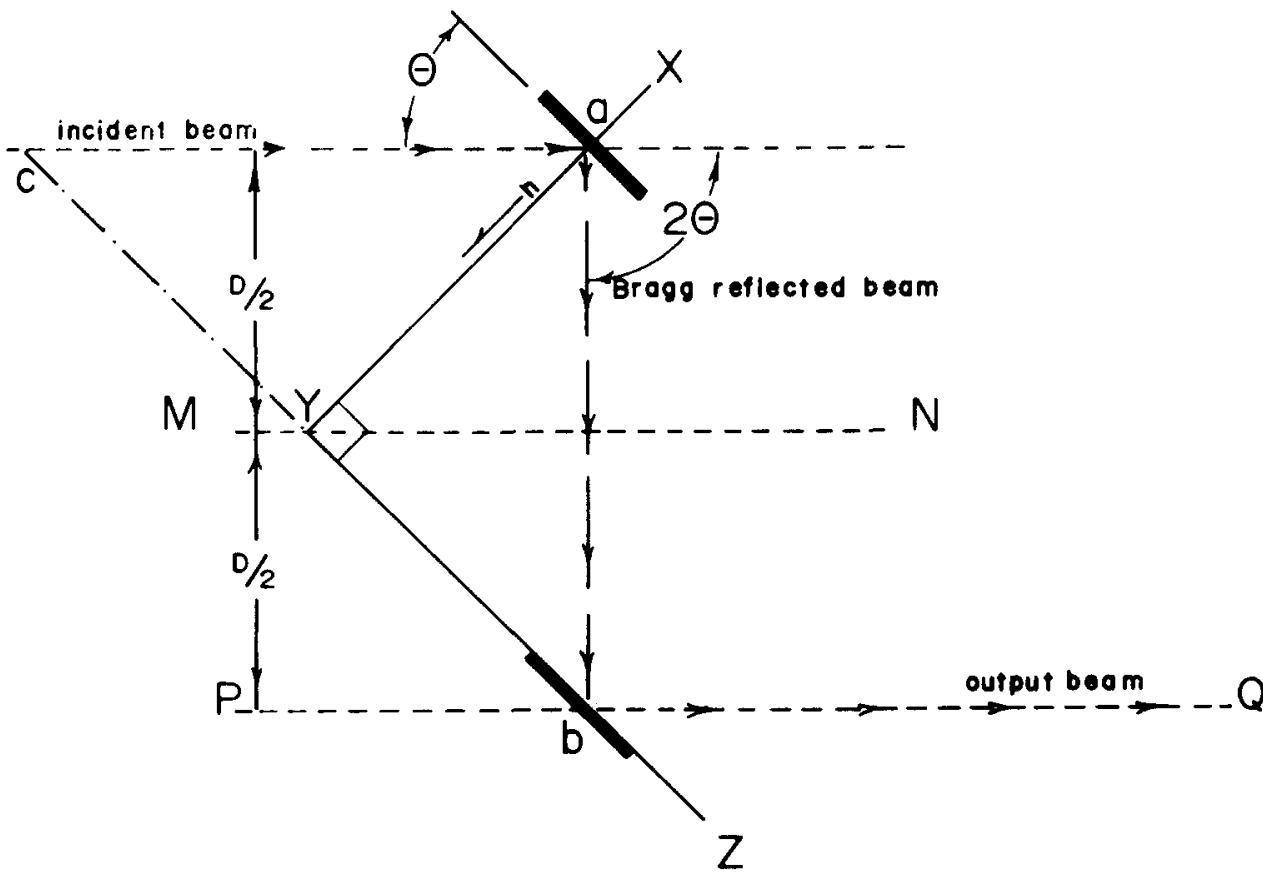

Fig. 2. Geometrical proof of monochromator action. Heavy solid lines at " $a$ " and " $b$ " show location of crystals. 
FIG. 3. Summary of motions required for operation of linkage. Bearing positions for a low-energy setting are drawn ting are dashed. in solid and those for a higher energy set-

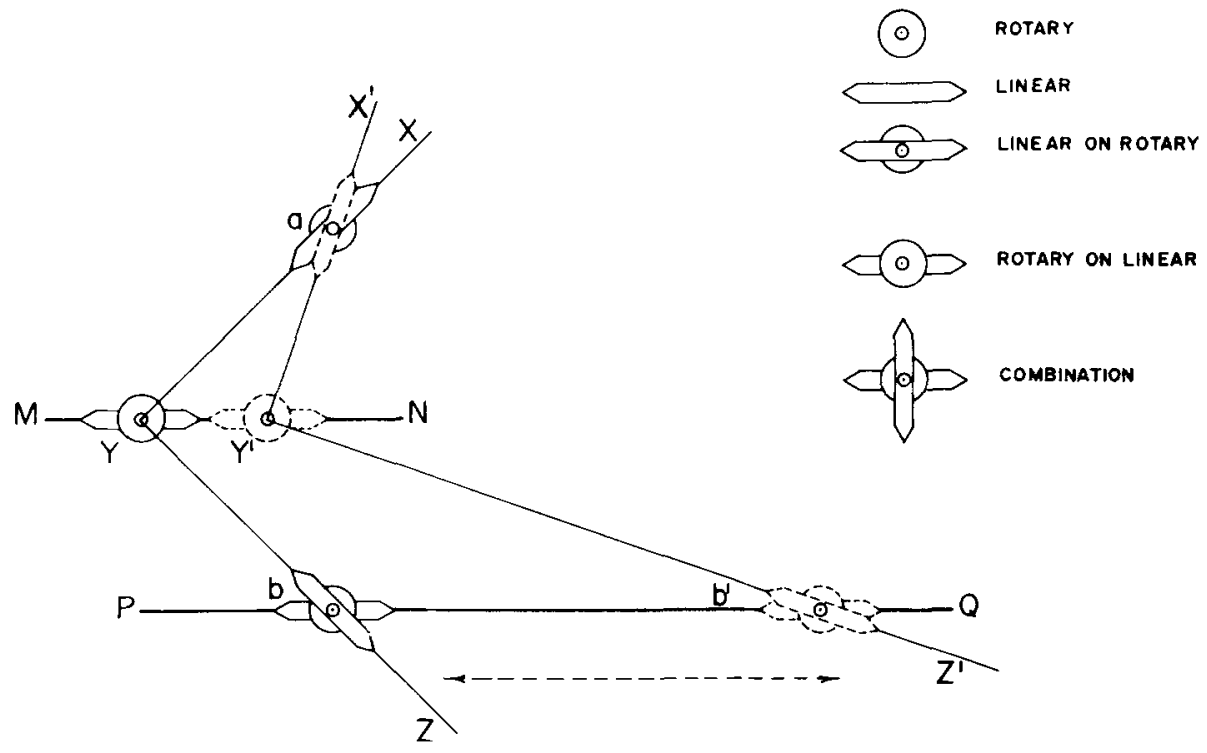

It is now possible to prove that regardless of the horizontal position of $Y$, i.e., regardless of the energy chosen, the beam reflected from " $a$ " will always intercept the bar $Y Z$ at the desired beam height. That is, the reflected beam, the bar $Y Z$ and the line $P Q$ will all intercept one another at a point. We call this point " $b$ " in the figures. To prove this assertion one may extend the line $Z Y$ to intercept the incident beam height at point $c$. The equality of incidence and reflection angles from the first crystal coupled with the congruence of triangles $Y a c$ and $Y a b$ yields the desired result.

So finally if the second crystal is mounted so as always to (1) be at a position " $b$ " (it will always intercept the beam selected by the first crystal), and (2) have its reflecting plane parallel to $Y Z$ (so that it will also be parallel to the planes of the first crystal), then the output beam be deflected along $P Q$ regardless of the energy chosen (regardless of the position of $Y$ along $M N$ ).

It is perhaps worthwhile to summarize the required motions for the mechanical linkage to work properly. This is done graphically in Fig. 3 where two possible settings of the linkage are shown, the one with right angle $X Y Z$ corresponding to choice of a lower energy, and $X^{\prime} Y^{\prime} Z^{\prime}$ to a higher energy $\mathrm{X}$-ray beam. In changing energy the rigid bar $Y X$ must slide through and rotate about point " $a$." The linear bearing through which $Y X$ slides must therefore be mounted on a rotary bearing whose axis is at " $a$ " perpendicular to the plane of the figure. The first crystal is mounted directly over the linear bearing at " $a$ " and perpendicular to $Y X$.

Another compound linear slide and rotary motion allows the apex $Y$ to translate along rigid $\operatorname{rod} M N$. This rod lies halfway between the incident beam height and the exit beam height. The latter coincides with the rigid bar $P Q$ on which the final bearing package slides. This combination contains two linear slide bearings joined by a rotary bearing. The second crystal mounts above the slide through which $Y Z$ passes and is oriented
FIG, 4. Modification of rigid right angle location.

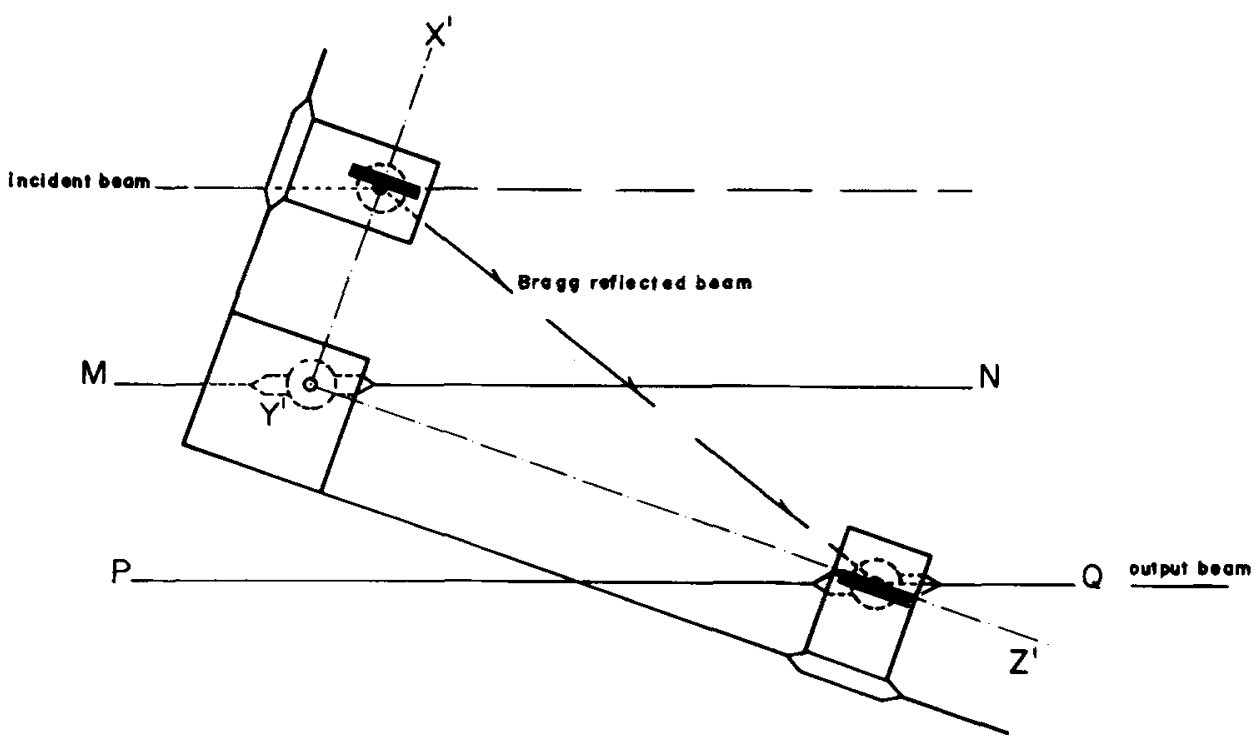



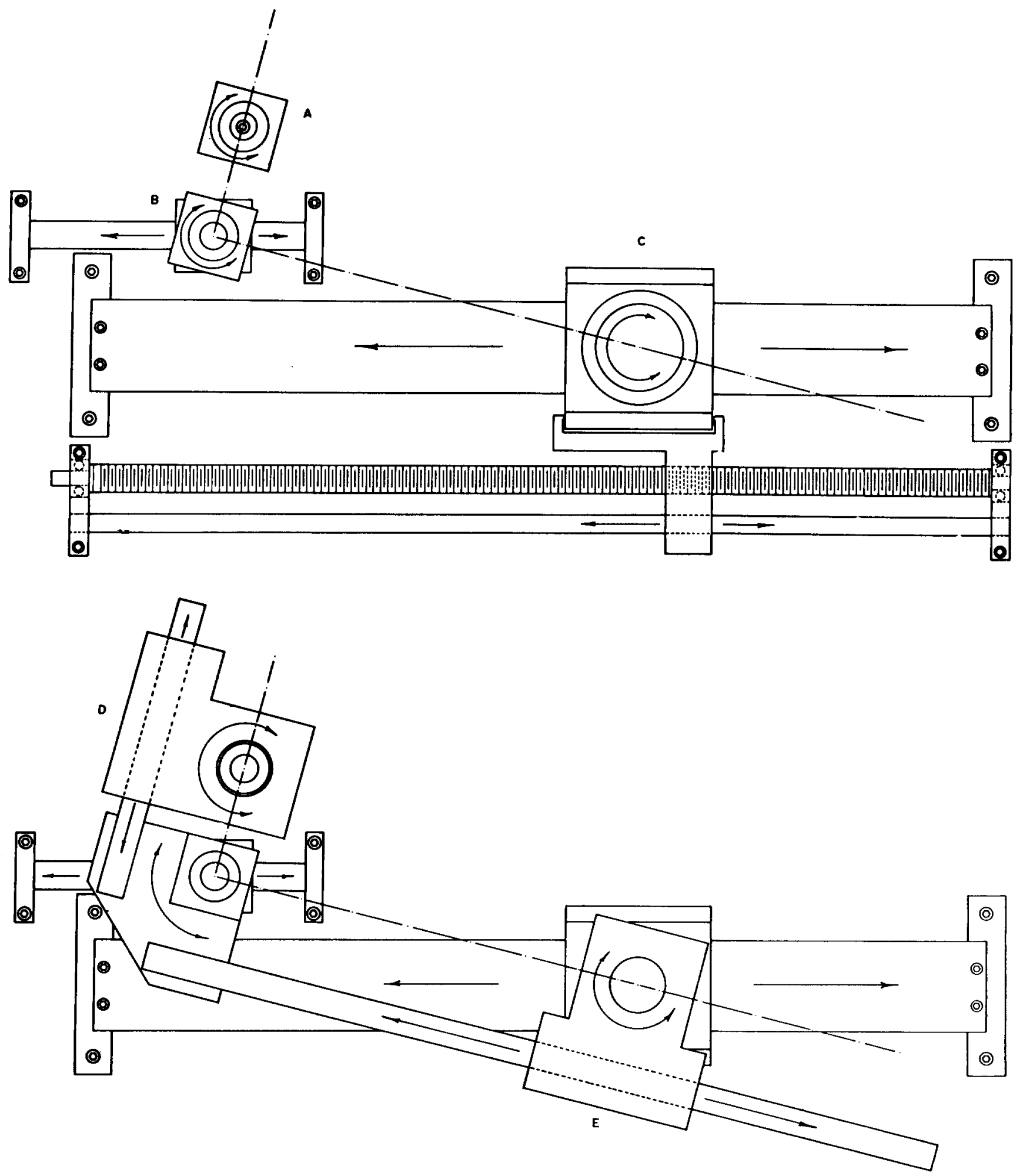

Fig. 5. Realization of linkage in successive stages of assembly using air bearings.

parallel to $Y Z$. (Figure 2 shows the orientation of crystals which are omitted from Fig. 3 for clarity in bearing illustration).

For reasons of convenience in construction we decided not to have the sliding right angle pass through the positions occupied by the crystals. Figure 4 shows how this can be accomplished without the right angle $X^{\prime} Y^{\prime} Z^{\prime}$ actually being realized by physical members.
The rectangular plates at the crystal position accommodate the corrector assembly to be described shortly.

\section{MECHANICAL REALIZATION}

The mechanical aspects of the monochromator were first studied using an inexpensive "mockup" based on ball bearings and Thompson circulating ball linear bear- 


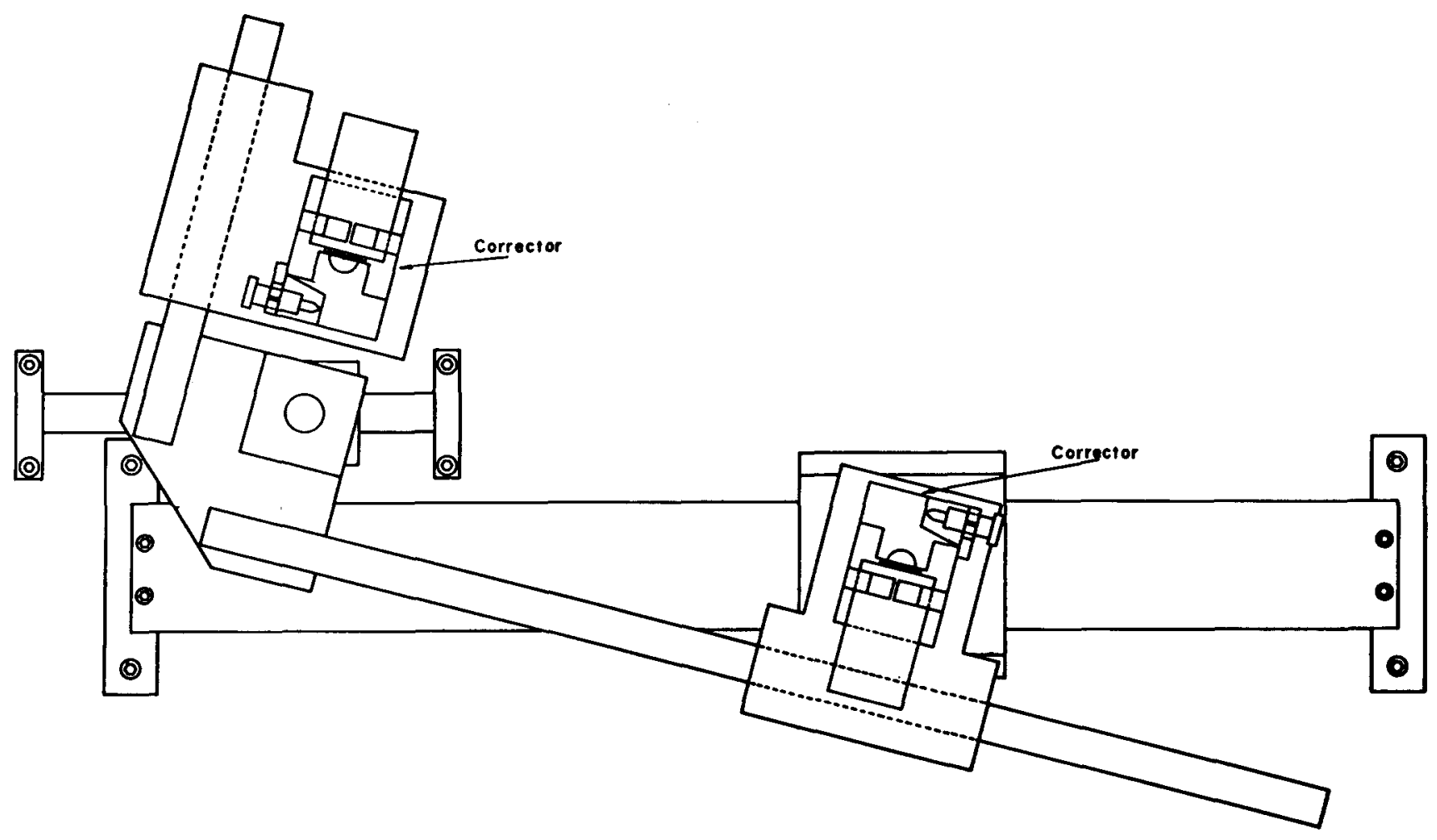

FIG. 5. (Continued)

ings. After some experimenting a design based totally on air bearings was constructed and tested.

Figures 5(a), (b), and (c) show the monochromator in successive stages of assembly. The first crystal air bearing assembly is indicated by " $A$ " in Fig. 5(a). This element consists of a stationary $3 / 4$ in. central steel rod serving as an axle for the surrounding air bushing (Dover LB-7) and pillow block enclosure which together are free to rotate as shown by the arrow. Motion perpendicular to the page is constrained by endcaps also working on the air bearing principle which results in the first element actually being an air thrust bearing.

The bearing package " $B$ " in Fig. 5(a) consists of another $3 / 4$ in. axle whose axis lies perpendicular to the plane of the figure, but in contrast to the similar piece at " $A$ " it is here mounted on the pillow block containing another air bushing that is free to translate horizontally on another steel rod. This motion is indicated by the straight arrows on the upper horizontal steel rod in the figure. Finishing up this element is another air bushing and pillow block assembly free to rotate about the axis perpendicular to the figure (curved arrows). Motion perpendicular to the page is constrained as at " $A$. ."

The second crystal air bearing assembly is labeled " $C$ " in Fig. 5(a). A large linear bearing package translates this assembly in the horizontal. It consists of a 24 in. long $2 \frac{1}{2} \times 1 \frac{1 / 4}{4}$ in. ground rectangular shaft on which a mated air bearing (Dover \#400B) slides as indicated by the horizontal arrows on the rectangular shaft. The energy selected from the synchrotron beam is determined ultimately by the horizontal position of this bearing. Energy control is therefore obtained through a lead-screw drive assembly shown at the bottom of Fig. 5(a). A rotary thrust bearing assembly (Dover Model 250) allows the final rotary motion, about an axis perpendicular to the figure, required by our linkage. This degree of freedom is again indicated by a curved arrow.

The next level of assembly is indicated in Fig. 5(b). Here the right angle member and its associated linear bearings (shown dotted) are shown mounted to the thrust bearing and pillow block assemblies previously described. The linear bearings at " $D$ " and " $E$ " in the figure are air bearings (Dover LB7) whose pillow blocks are mounted on plates that rotate about the first and second crystal positions by virtue of the rigid connections to the thrust bearings at those positions. The $3 / 4$ in. centerless ground rods that make up the physical right angle are rigidly connected together on a plate that rotates about the virtual right angle apex of Figs. 1, 2, and 3 . The now coupled rotary motions of all three of the above described plates are indicated by the curved arrows in Fig. 5(b).

The final level of assembly is indicated in Fig. 5(c). Here two new assemblies, which we call the correctors and which house the diffracting crystals, are shown mounted on the plates. The crystals are attached to the correctors over the positions indicated in Fig. 4. The final difference in height of the two crystals, and therefore the difference in height between incident and monochromated beams, was 6 in. in our design. This completes the mechanical realization of our linkage. The 


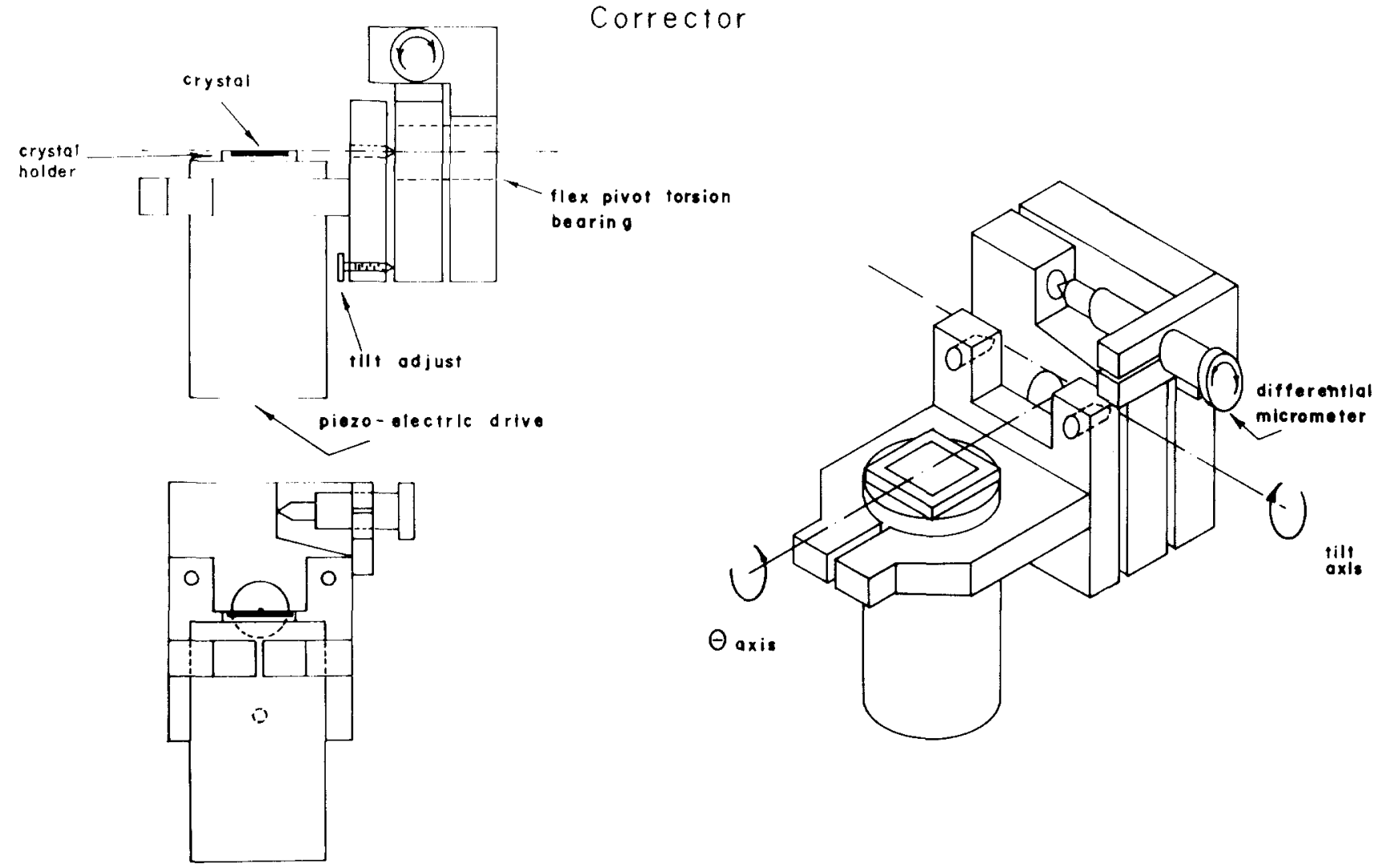

FIG. 6. Corrector assembly. Crystal is mounted on electronically controlled piezocrystal drive which in turn is mounted on a small goniometer stage controlled by a tilt adjust screw and a differential micrometer screw.

role of the corrector assembly is elaborated in the next section.

\section{THE CORRECTOR ASSEMBLY}

Preliminary adjustment of crystal angles and the ultimate fine angle feedback control of crystal parallelism requires an additional mechanical assembly we have dubbed the "corrector." The first job of the corrector is to allow the normal to the diffracting crystal planes to be made parallel to the bars $Y X$ and $Y Z$. Crystals can be cut and polished accurately to the order of a few tenths of a degree. This sets the scale for the range of angular correction anticipated in initial alignment. Due to the small angular emittance of the synchrotron source the alignment of the crystals through the axis perpendicular to the plane of the figures, the $\theta$ correction, is the most critical adjustment. The second adjustment is a tilt that rotates the crystal normal in and out of the plane of the previous figures. Figure 6 shows a drawing of the corrector assembly [see also Fig. 5(c)]. Since required angular adjustment range is minimal, Bendix flex pivots (torsion bearings) have been used for the bearings supplying the $\theta$ corrections. The angle is adjusted by a differential micrometer screw (Klinger Model \#385-034) on a tangent arm of $3 \mathrm{~cm}$. These micrometers can be controlled by dc motors and yield an angular range of $\sim 1^{\circ}$. In series with this is a fine electronically-controlled motion driven by a piezoelectric adjuster (Burleigh PZT 40) capable of 120 arcsec of angular control. The crystals mount directly on the PZT pusher in interchangeable mounts.

The second angular degree of freedom in the corrector is controlled by thumb screw adjustments on ball kinematic mounts (see Fig. 5).

\section{FEEDBACK ELECTRONICS}

A block diagram of the electronic feedback system integrated with the mechanical piezo controller is shown in Fig. 7. A high voltage operational amplifier capable of $0-1000 \mathrm{~V}$ output high slew rate and programability (Burleigh PZ 90) directly drives the piezoelectric element. This drive signal contains a dc bias $(0-1000 \mathrm{~V})$ that sets the mean angle of the second crystal and a modulation signal at $100 \mathrm{~Hz}$ and approximately $0.1-1 \mathrm{~V}$ peak to peak that results in a small ac component in $x$-ray output signal. The output beam passes through a transmission ionization chamber whose signal is amplified by a (Keithly 427) current amplifier with a gain in the neighborhood of $10^{*} \mathrm{~V} / \mathrm{A}$ and frequency response commensurate with the ac probe content of the signal. The voltage output from this stage drives a (P.A.R. Model $124)$ lock-in amplifier. This lock-in amplifier is referenced to a sine wave oscillator at $100 \mathrm{~Hz}$ (Hp Model 3310-B) that also provides the angle modulation signal to the high voltage operational amplifier previously described. The phase sensitive dc output of the lock in then directly drives the operational amplifier input to adjust the mean angle for minimum $100 \mathrm{~Hz}$ content in the output $\mathrm{x}$-ray 
FIG. 7. Electronic feedback system. Ion chamber monitors output beam intensity and originates feedback signal that ultimately controls piezocrystal setting.

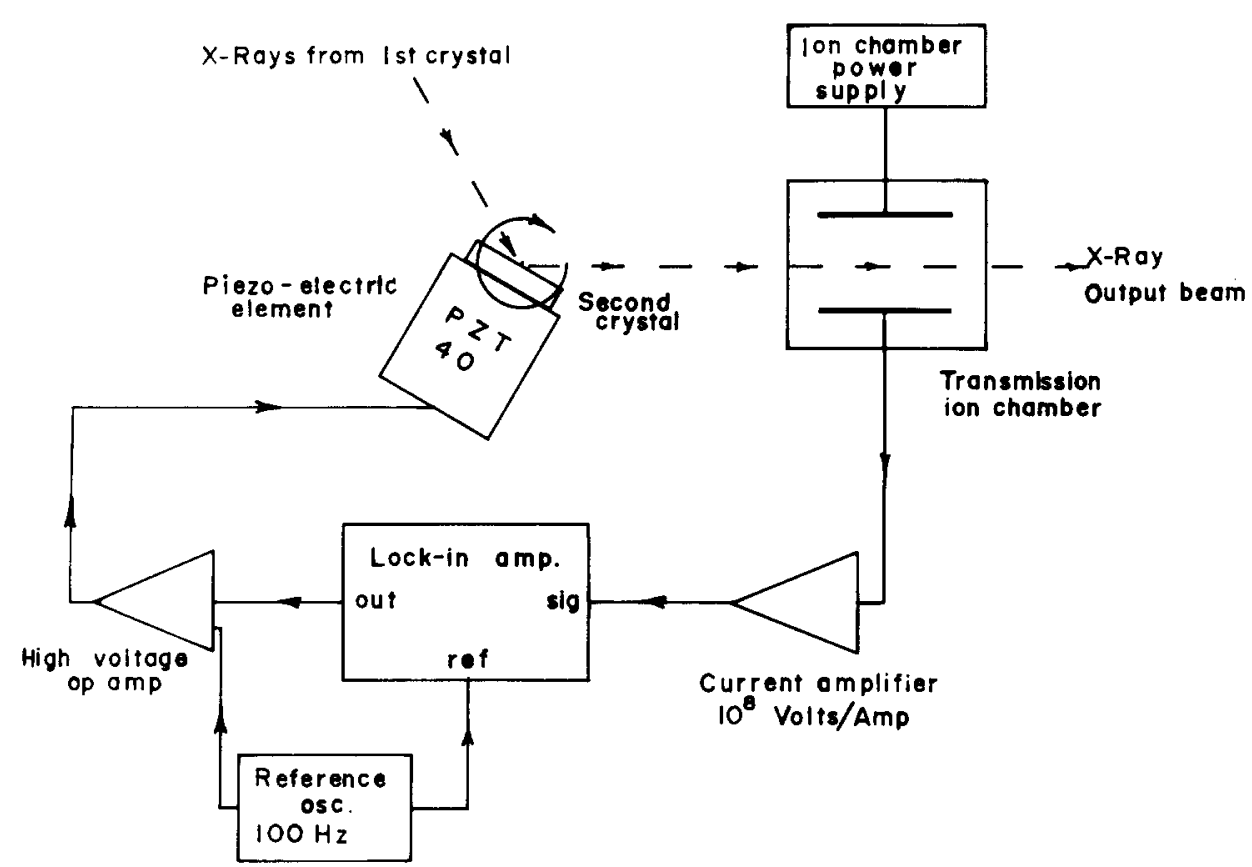

beam. That is, the feedback stabilized system stays at the point of zero first derivatives on the top of the double crystal reflection curve. We note here that it is possible to adjust a feedback system of this type to sit on the side of the reflection curve for improved harmonic suppression. This modification of our apparatus will be discussed elsewhere.

\section{MONOCHROMATOR ALIGNMENT AND OPERATION}

The assembled monochromator was mounted on a steel table with adjustable height and level controls. For first and second crystals, silicon was chosen with 220 reflecting planes approximately parallel to the surface. The reflecting surface area was $4 \mathrm{~cm}^{2}$ and crystal thickness was $5 \mathrm{~mm}$. The surface was Syton polished to provide an optical mirror surface. Preliminary alignment was performed with a $\mathrm{HeNe}$ alignment laser simulating the synchrotron beam. An arbitrary energy position was selected on the monochromator and the " $\theta$ " motion on the first crystal corrector is adjusted so the reflected light beam impinges on the second crystal at its center. The laser beam was then made to enter the monochromator from the exit beam side onto the second crystal. The " $\theta$ " correction on this crystal was then adjusted to make the reflected beam strike the first crystal at its center. During these two previous adjustments the corrector tilt controls would also be set so crystals are approximately parallel. Proper alignment at this stage allowed us to pressurize the monochromator air bearings and scan without feedback through the energy range of the instrument with laser beam direction changing less than or of order 30 arcseconds. This completed the preliminary alignment and testing.

Unfortunately it is not possible to guarantee exacting parallelism between the polished surface and crystal planes so a secondary alignment must now be done with the synchrotron beam. This was accomplished most easily by the following procedure. Again at an arbitrary energy a beam was reflected from the first crystal and a photograph of its position on the second crystal was taken. The first crystal corrector was adjusted to position the reflected beam at the center of the second crystal. A detector ( $\mathrm{NaI}$ scintillator) and phototube were then placed at the monochromator output slit and the second crystal corrector " $\theta$ " motion was scanned for the second reflection. After finding the reflection a transmission ion chamber replaced the $\mathrm{NaI}$ counter and a preliminary scan of the rocking curve was taken by scanning the second crystal piezo drive. At this point the expected natural rocking curve width was observed. The feedback electronics was then actuated and the system was scanned in energy from $4 \frac{1}{2}-17 \mathrm{keV}$. This was accomplished by connecting a small dc motor with appropriate gear reduction to the lead screw assembly described in connection with Fig. 5(a). For energy

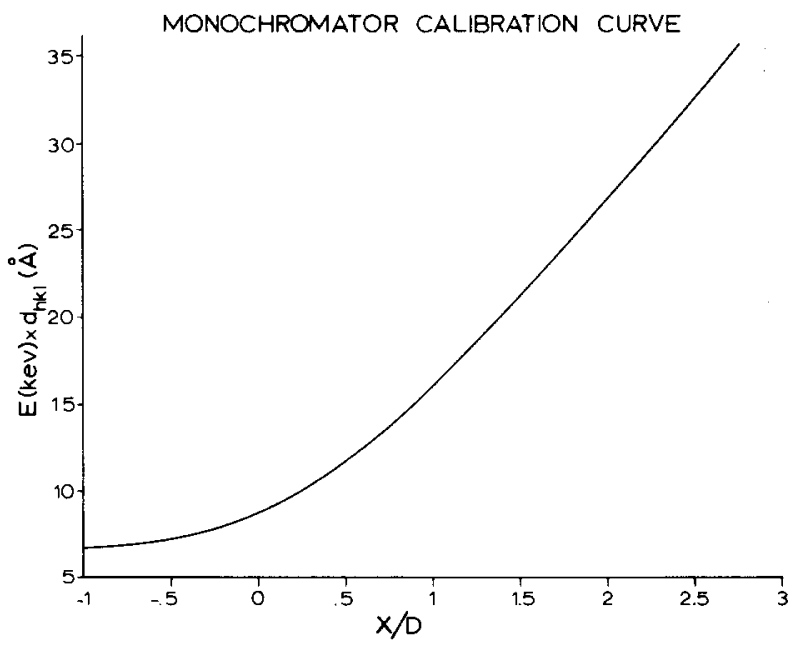

FIG. 8. Monochromator calibration curve. 
readout a Heidenhain Posecon linear position readout system with an accuracy of 0.5 mils over 18 inches was also connected to the lead screw. From the change in dc output of the high voltage piezo driver op amp with scan energy, it was also clear that a maximum of approximately 30 arcseconds of corrective angular adjustment was being supplied by the feedback system. This is consistent with the expected needs deduced from the aforementioned laser beam measurements. The feedback system worked well over the entire energy range with a $100 \mathrm{~Hz}$ component of $-0.1 \%-1 \%$ of the total $\mathrm{x}$-ray signal which was approximately $1 \mathrm{~V}$ at the output of the current amplifier. Typical values of closed loop gain were $10-100$ and closed loop time constant were $\sim 0.1 \mathrm{~s}$.

Figure 8 shows a calibration curve enabling conversion of the linear position readout to $\mathrm{x}$-ray energy in $\mathrm{keV}$ based on the formula

$$
E d_{h k l}=\frac{6.19}{\sin \left(\frac{1}{2} \tan ^{-1} D / \chi\right)} .
$$

Here $E$ is the beam energy in $\mathrm{keV}, D_{h k l}$ is the Miller plane spacing in Angstroms for the diffracting crystals, $D$ is the offset in beam height designed into the monochromator, and $\chi$ is the linear position readout of the second crystal. The origin for $\chi$ is the position where the second crystal lies directly below the first crystal. It is seen from Fig. 8 that $E d_{h k l}$ vs $\chi / D$ becomes linear towards high energy. At high energy with a $\mathrm{Si}(220)$ diffracting crystal the energy dispersion of our design ( $L=6 \mathrm{in}$.) is about 1 $\mathrm{eV} / 0.001$ in. in $\chi$.

\section{FINAL COMMENTS}

There are several possible ways the design of the present monochromator may be modified. A more compact instrument would result by diminishing the value of $D$. Higher energies can be achieved by simply extending the right angle bar that passes through the second crystal and either extending or just moving the second crystal horizontal slide further out.

For many experiments, control over harmonic content in the output beam is essential. Several methods are available to reduce the harmonics. Total external reflection $\mathrm{x}$-ray mirrors can be conveniently used either before or after the monochromator with incidence angles chosen so the harmonic beams lie above their critical angles. The absence of output beam height variation with energy allows the possibility of even having such harmonic suppression capability downstream at the experiments. On the other hand the piezoelectric feedback system previously described can be modified to stabilize on the side of a rocking curve where harmonic content is reduced. This can be accomplished by stabilizing on second derivative signals at twice the piezo driving frequency.

When an absorption or scattering in the monochromator presents a problem, the air bearings may be driven by compressed helium in a closed system. For operation at very low energies the monochromator must operate in a vacuum beam line. The air-bearing realization of our mechanism is then unsatisfactory and we are currently looking for an alternative approach to solve this problem.

The monochromator described in this article has been aligned and tested at the Cornell High Energy Synchrotron Source at Cornell University, where it currently resides. It has been used in Compton scattering, $x$-ray topography, x-ray interferometry, and standing wave experiments with success.

\section{ACKNOWLEDGMENTS}

We would like to thank W. L. Brown of Bell Labs for his encouragement and support for this project and Prof. B. Batterman for providing access to the C.H.E.S.S. facility at an early stage. We also acknowledge Brian Kincaid of Bell Labs for valuable discussion.

\footnotetext{
Present address: National Bureau of Standards, Washington, D.C. 20234.

I Sunchrotron Radiation-Techniques and Applications, edited by C. Kunz (Springer-Verlag, New York, Heidelberg, Berlin, 1979), pp. 122-132.

- M. Lemonnier, O. Collet, C. Depautex, J. Esteva, D. Raous, Nucl. Instrum. Methods 152, 109 (1978).
} 